\title{
A numerical study on magnetic polarity transition in an MHD dynamo model
}

\author{
Futoshi Takahashi, Masaki Matsushima, and Yoshimori Honkura \\ Department of Earth and Planetary Sciences, Tokyo Institute of Technology, 2-12-1 Ookayama, Meguro-ku, Tokyo 152-8551, Japan
}

(Received September 30, 2006; Revised June 1, 2007; Accepted June 1, 2007; Online published July 20, 2007)

\begin{abstract}
Magnetic polarity transitions in a Takahashi-Matsushima-Honkura dynamo model are analyzed. Distinctive differences in behavior of the axisymmetric poloidal magnetic field are found among a polarity reversal and excursions, including short polarity events. At the beginning of magnetic polarity transitions, the magnetic field with the reversed polarity is generated by anti-cyclonic convection columns deep within the outer core. In the case of excursion, it is soon advected by the radial flow toward a shallow interior of the core, and the transition can be detected at the core surface. However, the same process retrieves the original polarity from the deep interior, and the reversed field eventually vanishes. In the case of polarity reversal, on the other hand, the reversed polarity field is persistently generated deep within the core. It is then advected toward a shallow interior of the core, while the generation process of the reversed field occurs successively. The reversed polarity field near the core surface is collected by the downwelling flow associated with convection columns, as is the case for the original polarity field. The polarity reversal is completed by the advection process, the duration of which is consistent with the flow speed in the core.
\end{abstract}

Key words: Dynamo, magnetic polarity transition, advection, stretching.

\section{Introduction}

The mechanism of the polarity reversal of the geomagnetic field is one of the unsolved problems in geophysics, and it has been investigated through magnetic measurements of paleomagnetic samples and numerical simulations of magnetohydrodynamic (MHD) processes in rapidly rotating spherical shells. Paleomagnetic studies have clarified some characteristics of reversals, such as the duration of polarity transition (4,000-5,000 yr), its site dependence (Clement, 2004), and changes in the intensity and direction of the field during transition (e.g. Merrill et al., 1996). Excursions are the second type of geomagnetic feature identified with large polar swings away from the geographic pole that are possibly accompanied by a relatively small dipole moment (e.g. Mochizuki et al., 2006). Although paleomagnetic studies have provided information on the real geomagnetic field in the past, the paleomagnetic fields can be sampled only around the Earth's surface or, at most, at the core-mantle boundary (CMB) through downward continuation of the geomagnetic potential field. Therefore, we must rely on numerical simulations of MHD dynamo models to understand the process of polarity reversal and excursion as well as that of geomagnetic field generation deep inside the core. For example, excursions have often been subjected to the idea that they are aborted reversals prohibited by the electrically conducting inner core (Gubbins, 1999), but the dynamo process is found to be only slightly affected by the inner core of the present size through dynamo simulations with the conducting and insulating inner core (Wicht, 2002).

Copyright (c) The Society of Geomagnetism and Earth, Planetary and Space Sciences (SGEPSS); The Seismological Society of Japan; The Volcanological Society of Japan; The Geodetic Society of Japan; The Japanese Society for Planetary Sciences; TERRAPUB.
In reality, many numerical dynamo models have demonstrated that polarity reversals can occur spontaneously by the MHD process in the core (e.g. Kono and Roberts, 2002). After a reversing dynamo model was first obtained by Glatzmaier and Roberts (1995), many numerical dynamo models showing polarity reversals have been examined from paleomagnetic and statistical points of view (Coe et al., 2000; McMillan et al., 2001; Kutzner and Christensen, 2004; Bouligand et al., 2005; Wicht, 2005). It was found that they are consistent with some of the evidence observed during polarity reversals, as mentioned above. However, most numerical models have focused on analyzing the magnetic field at radial levels representing the CMB or Earth's surface in order to compare with paleomagnetic results, regardless of the fact that results have been obtained in the entire region of the fluid core. Accordingly, it is strongly required to examine dynamo action in the core to understand the essence of polarity reversals.

Using a 2.5-D dynamo model, in which the axisymmetric component and only one non-axisymmetric mode are included, Sarson and Jones (1999) suggested that the polarity reversal is triggered by intermittent upwelling flow inside the tangent cylinder (TC), an imaginary cylinder coaxial with the rotation axis circumscribing the inner core at the equator. Li et al. (2002) obtained irregular polarity reversals in the Kageyama-Sato dynamo model (Kageyama et al., 1995) and suggested that the occurrence of polarity reversals is caused by the equatorially asymmetric convection. Wicht and Olson (2004) closely investigated the reversal process inside the core using their dynamo model and found that the reversed magnetic field is generated in the high-latitude helical plumes. It should be noted that they adopted relatively tractable parameter values for the analy- 
sis with their dynamo model. Although this was a judicious choice to extract fundamental processes of the geomagnetic polarity reversal, it means that parameter values adopted in their model are far from those of the real Earth's core. Here we present the analysis of a reversal mechanism in a significantly more realistic dynamo model.

Takahashi et al. (2005) recently obtained a dynamo model dynamically similar to the geodynamo; that is, the viscosity of core fluid is sufficiently small and the core is in the so-called quasi-Taylor state. The model also shows some polarity reversals and excursions, which provides a good opportunity to examine the reversal processes and compare them to those of previous studies. In this paper, we analyze the dynamo model to gain an understanding of the process during transitional periods and to search for any indication of the internal process of the polarity reversal and excursion.

\section{Mathematical Model and Numerical Method}

Since mathematical formulation and the numerical method of our dynamo model have been described in our previous papers (Takahashi et al., 2001, 2003; Takahashi and Matsushima, 2005), we only summarize them here. We model a three-dimensional, time-dependent MHD dynamo model which generates magnetic field by thermal convection in a rotating spherical shell with the angular velocity $\Omega \hat{\mathbf{z}}$ in spherical coordinate system $(r, \theta, \phi), \hat{\mathbf{z}}$ being the unit vector in the $z$-direction. The spherical shell is filled with an electrically conducting Boussinesq fluid. Convection is driven by imposing the superadiabatic temperature contrast $\Delta T$ between the inner and outer boundaries.

The radius of the inner core, $r_{i}$, is 0.35 -fold of the outer core radius, $r_{o}$. The solid inner core is electrically insulating, which hardly affects the temporal evolution of the magnetic field due to its small size (Wicht, 2002). The electrically insulating conditions as well as no-slip and isothermal conditions are then applied to the inner and outer boundaries.

We solve the Navier-Stokes equation, the magnetic induction equation, and the heat transport equation together with the equations of continuity for the velocity and magnetic fields. They are non-dimensionalized using the following scalings: the thickness of the spherical shell $L=$ $r_{o}-r_{i}$ for the length, $L^{2} / \nu$ for the time, $v / L$ for the velocity, $\Delta T$ for the temperature, and $(2 \rho \mu \eta \Omega)^{1 / 2}$ for the magnetic field, where $v, \rho, \mu$, and $\eta$ are the kinematic viscosity, the density of the fluid, the magnetic permeability in free space, and the magnetic diffusivity, respectively. The governing equations in non-dimensional form are given as

$$
\begin{gathered}
E\left(\frac{\partial \mathbf{u}}{\partial t}+\mathbf{u} \cdot \nabla \mathbf{u}-\nabla^{2} \mathbf{u}\right)=-\nabla p+\mathbf{u} \times \hat{\mathbf{z}} \\
+\operatorname{RaE} \operatorname{Pr}^{-1} \Theta \frac{\mathbf{r}}{r_{o}}+P m^{-1}(\nabla \times \mathbf{B}) \times \mathbf{B}, \\
\frac{\partial \mathbf{B}}{\partial t}=P m^{-1} \nabla^{2} \mathbf{B}+\nabla \times(\mathbf{u} \times \mathbf{B}), \\
\frac{\partial \Theta}{\partial t}=\operatorname{Pr}^{-1} \nabla^{2} \Theta-\mathbf{u} \cdot \nabla\left(\Theta+T_{s}\right),
\end{gathered}
$$

$$
\nabla \cdot(\mathbf{u}, \mathbf{B})=0
$$

where $\mathbf{u}, \mathbf{B}, p$, and $\Theta$ are the velocity field, the magnetic field, the reduced pressure, and the temperature deviation from the stationary spherically symmetric temperature field obeying $\nabla^{2} T_{s}=0$, respectively.

The non-dimensional parameters that appear are the Ekman number, $E=v / 2 \Omega L^{2}$, the Prandtl number, $\operatorname{Pr}=\nu / \kappa$, the Rayleigh number, $R a=\alpha g_{o} \Delta T L^{3} / \nu \kappa$, and the magnetic Prandtl number, $P m=v / \eta$, where $\kappa$ is the thermal diffusivity, $\alpha$ the thermal expansion rate, and $g_{o}$ the gravitational acceleration rate at the outer boundary. Note that non-dimensional parameters are defined using the shell thickness in this paper, whereas Takahashi et al. (2005) use the outer core radius, $r_{o}$, as the length scale. The relations between $E$ and $R a$ and those in Takahashi et al. (2005), $E^{*}$ and $R a^{*}$, are given by $E^{*}=r_{o}{ }^{-2} E$ and $R a^{*}=r_{i} r_{o}{ }^{3} R a$, respectively.

We use the general decomposition of the divergence-free velocity and magnetic fields into the poloidal and toroidal parts:

$$
\begin{aligned}
& \mathbf{u}=\mathbf{u}_{P}+\mathbf{u}_{T}=\nabla \times \nabla \times(v \hat{\mathbf{r}})+\nabla \times(w \hat{\mathbf{r}}), \\
& \mathbf{B}=\mathbf{B}_{P}+\mathbf{B}_{T}=\nabla \times \nabla \times(g \hat{\mathbf{r}})+\nabla \times(h \hat{\mathbf{r}}) .
\end{aligned}
$$

Subscripts $P$ and $T$ denote the poloidal and toroidal parts, $\hat{\mathbf{r}}$ denotes the radial unit vector, $v$ and $w$ are the poloidal and toroidal scalar functions of the velocity field, and $g$ and $h$ are the poloidal and toroidal scalar functions of the magnetic field. All variables are expanded in terms of spherical harmonics in the angular directions. For instance, the scalar function of the poloidal magnetic field is represented as

$$
g(r, \theta, \phi, t)=\sum_{\ell, m} g_{\ell}^{m}(r, t) Y_{\ell}^{m}(\theta, \phi),
$$

where $Y_{\ell}^{m}$ is a fully normalized spherical harmonic function of degree $\ell$ and order $m$. Other scalar variables as well as $\Theta$ and $p$ are expanded in the same way.

We employ a pseudo-spectral method to solve Eqs. (1)(3) and adopt a finite difference method to evaluate derivatives in the radial direction. Spherical harmonic expansion is truncated at degree $\ell_{\max }=191$, and 150 radial grid points are located on the points defined as

$$
\begin{array}{r}
r_{j}=0.5\left(r_{o}+r_{i}-\left(r_{o}-r_{i}\right) \cos \left(\pi j / N_{r}\right)\right), \\
0 \leq j \leq N_{r}=149 .
\end{array}
$$

\section{Dynamo Model}

The values of model parameters are $E=10^{-5}, R a=$ $8 \times 10^{7} \sim 17 R a_{c}, P r=1$ and $P m=0.5$. The numerical simulation was run for 2.2 magnetic diffusion time. $R a_{c}$ is the critical Rayleigh number for the onset of non-magnetic thermal convection. This model shows an obvious polarity reversal and is thus selected for examination in this study. Analyses are made of the result after one magnetic diffusion time passed. The magnetic Reynolds number, $R m=L u / \eta$, the Elsasser number, $\Lambda=B^{2} / 2 \rho \mu \eta \Omega$, and the Nusselt number at the outer boundary, $N u$, where $u$ is the typical velocity and $B$ is the typical magnetic field, are calculated for time-averaged properties of the flow, the magnetic field, 
and the heat transfer. They are summarized as follows: $\mathrm{Rm}$ $=156, \Lambda=0.11$, and $N u=5.39$ (Takahashi et al., 2007). $R m$ and $\Lambda$ are somewhat lower than the expected Earth-like values, while they are in agreement with scaling laws by Christensen and Aubert (2006). Note that the Lorentz number $(L o=B / \sqrt{\rho \mu} \Omega L)$ - and not the Elsasser number-is used for magnetic field scaling in Christensen and Aubert (2006). Supercriticality of convection in this model seems to fall into the dipolar regime in Kutzner and Christensen (2002) and Christensen and Aubert (2006). Also, this dynamo model is found to lie in the dipolar regime according to Olson and Christensen (2006); the boundary between the dipolar and multipolar regimes is given by the local Rossby number, $R o_{\ell} \sim 0.1$, where $R o_{\ell}=R o \ell_{u} / \pi, R o=u / \Omega L$ is the global Rossby number, $\ell_{u}$ is the weighted average of $\ell$ based on the kinetic energy spectrum, and $R o_{\ell} \sim 0.07$ in this dynamo model. This difference may arise from different conditions between the models, such as longitudinal symmetry.

\subsection{Time series of magnetic polarity}

In order to identify excursions and to distinguish them from reversals, we adopt definitions commonly used in paleomagnetism. When deviation of the magnetic pole from the geographic north or south pole exceeds the threshold value, $45^{\circ}$, it is considered that an excursion or a reversal sets in. If the magnetic pole returns close to the same geographic pole where it started, we treat the variation as an excursion, irrespectively of whether the magnetic pole ever came closer than $45^{\circ}$ to the other geographic pole. Duration of a reversal or an excursion is determined as a time span during which the magnetic pole deviation stays beyond the threshold value $\left(\geq 45^{\circ}\right)$. Although the strength of a dipole moment can also be used as an additional criterion in numerical dynamo models (Wicht, 2005), we rely on the location of the magnetic pole here, following the definition in paleomagnetism. Two stable polarity periods are separated by a reversal, and excursions are permitted during these periods, since the excursion is a rather frequent event (Singer et al., 2002). In fact, Oda et al. (2004) report as many as 20 excursions in the Brunhes chron. For example, three subsequent excursions occurred within a short interval between 30-60 ka; Laschamp excursion ( $40 \pm 2 \mathrm{ka}$, Guillou et al., 2004) and two Auckland excursions ( $29 \pm 3 \mathrm{ka}$ and $53 \pm 4$ ka, Mochizuki et al., 2004, 2007).

Figure 1 shows the time sequence of the polarity and magnetic energy fluctuations. The top panel in Fig. 1 exhibits the colatitude of the pole for the magnetic dipole moment. Its temporal behavior is chaotic, but we can find one reversal and six excursions, three of which comprise polarity change, during about one magnetic diffusion time. Hereafter, we call these time spans for the reversal and excursions transitional periods, labeled as R1 and E1-E6, as shown in Fig. 1. The time scale of each event ranges from 2,000 to 6,000 years, where the magnetic diffusion time is 170,000 years, assuming the magnetic diffusivity of $\eta=1 \mathrm{~m}^{2} / \mathrm{s}$. Such a time scale is comparable to the durations of polarity reversals and excursions found in the geomagnetic field (Merrill et al., 1996). In R1, the polarity is reversed around $t=0.75,2,000$ years after the start of the reversal. The short excursion E2 found prior to rever-
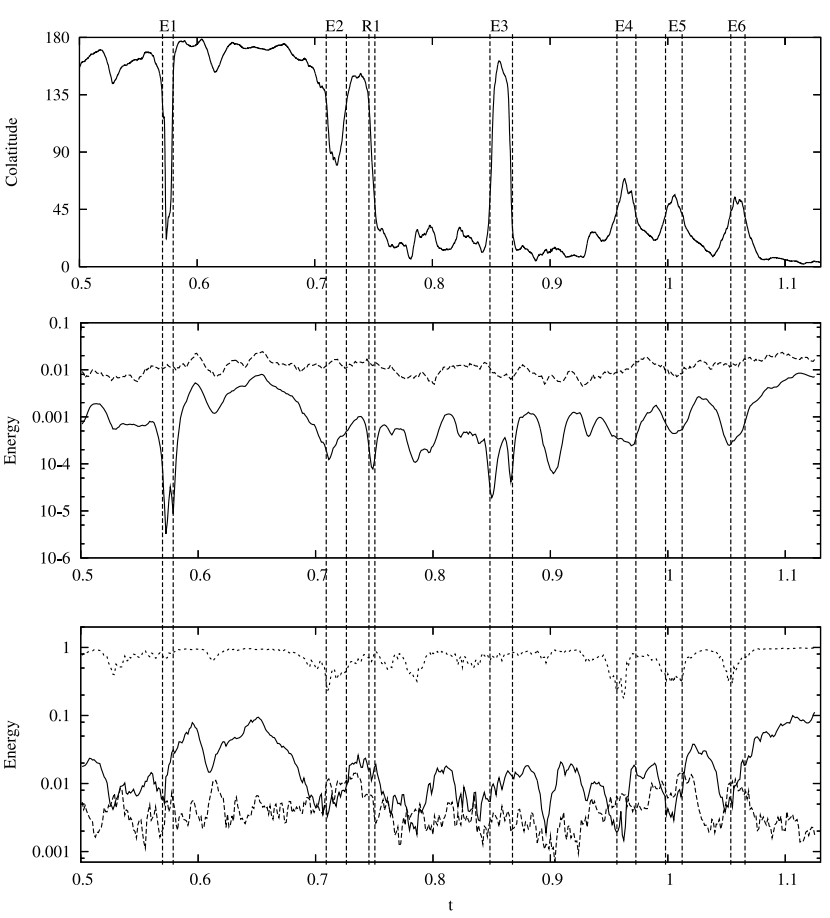

Fig. 1. Time series of dynamo simulation. The top panel shows the co-latitude of the pole for the magnetic dipole moment; the middle panel shows the magnetic energy of the dipole component (solid line) and of the non-dipole components (dashed line) at the CMB; the bottom panel shows the magnetic energy of the axial dipole (solid line), the equatorial dipole (dashed line) components in the core, and the relative contribution of the axial dipole component to the total dipole (thin dashed line). Each event is depicted by the dashed vertical lines.

sal R1 could be interpreted as a precursor rather than an independent event. Similar features are found prior to the Matuyama-Brunhes polarity change (Brown et al., 2004). Thus, it may be better to think of E2 and R1 as one reversal.

The middle panel in Fig. 1 shows the time sequence of the energy of the dipole and non-dipole components at the CMB. Although the transitional periods correlate well with the minima of the dipole, not all dipole minima lead to transitional configurations, indicating that both axial and equatorial dipole components are low. In other words, the intensity drop at the CMB itself does not always indicate the beginning of a transitional period. The same argument is true to some extent inside the core, as shown at the bottom of Fig. 1 where the time sequence of the energy of the axial and equatorial dipoles within the core are given. Events E2, E4, E5, and E6 correspond to minima of magnetic energy for the axial dipole, which is comparable to or even smaller than magnetic energy for the equatorial dipole component. On the other hand, the fraction of the axial dipole to the total dipole within the core does not change significantly during events E1 and E3. This fact does not mean that both the equatorial and the axial dipole components are weak during E1 and E3, as found in the bottom of Fig. 1. It will turn out below that these events originate in the shallow interior of the core.

The structure of the magnetic field, including the dipole polarity, is best described in the meridional cross section of the axisymmetric component. Its time evolutions during transitional periods, R1, E1 and E4, are exhibited in 


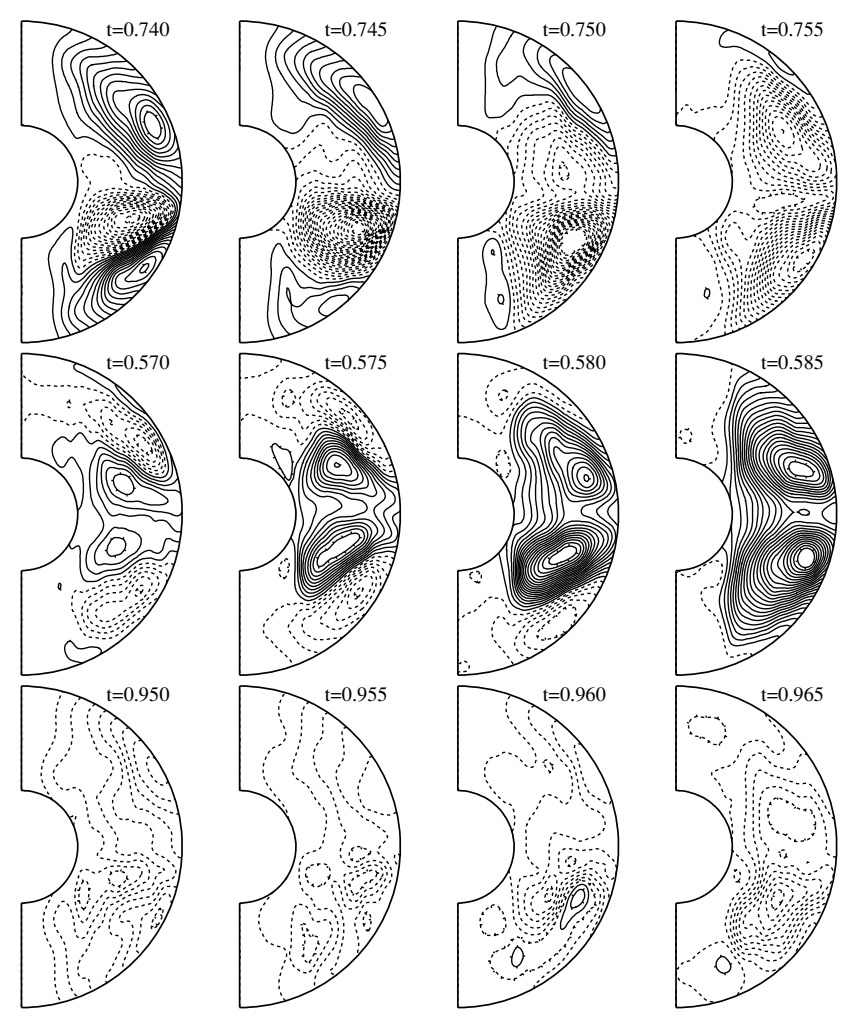

Fig. 2. Time evolutions of the axisymmetric components of the magnetic lines of force in a meridional section during sequences of the events R1 (top), E1 (middle), and E4 (bottom). Solid (dashed) lines denote the anti-clockwise (clockwise) field. Contour interval is 0.003 for all plots.

Fig. 2, in which behaviors of the magnetic field specific to these three periods are clearly seen. As for R1, the deeprooted magnetic field with the reversed polarity grows in the southern hemisphere at $t=0.740$. During this process, the reversed magnetic field is transported toward the northern hemisphere by advection between $t=0.745-0.750$. Finally, the polarity changes by the fully developed magnetic field with the reversed polarity in both hemispheres at $t=$ 0.755 .

We now analyze event E1. The reversed magnetic field appears in both hemispheres in the shallow interior of the core, and it grows somewhat at $t=0.570$. The magnetic field with the original polarity is retrieved from the deep, pushing the reversed field towards the outer boundary. The magnetic polarity then seems to be reversed at the core surface at $t=0.575$. However, the reversed magnetic field is located only at the shallower interior of the core. The magnetic field with the original polarity grows from the deep interior and prevails over that of the reversed polarity at $t=0.580$. Eventually, the polarity reverses again back to the original sign at $t=0.585$. Next, let us look at the E4 event. As in the E1 event, the weak reversed field appears in the shallow interior of the core, but only in the southern hemisphere at $t=0.960$. This phenomenon can be observed as an excursion without polarity change outside the core. The adjacent original field prevails once again in the southern hemisphere, and the excursion terminates at $t$ $=0.965$. The panels illustrating E4 in Fig. 2 show a much weaker magnetic field than for R1 or E1. The reason for
Table 1. Summary of the characteristics of the reversed field that causes the polarity transition. E2 is not included.

\begin{tabular}{ccccc}
\hline & Location & Hemisphere & Polarity change & Duration (kyear) \\
\hline R1 & deep & one then both & yes & 2.1 \\
E1, E3 & shallow & both & yes & $3.1-6.4$ \\
E4-E6 & shallow & one & no & $3.4-5.1$ \\
\hline
\end{tabular}

this is that the axial dipole field remains generally weak during events E4-E6. This feature is concordant with a recent paleomagnetic study on excursions (Mochizuki et al., 2007).

We summarize the characteristics of the reversed magnetic field that causes a polarity transition in Table 1 . In the case of excursions, the reversed field generated in the deep interior of the core soon moves toward the shallow interior, and disappears. In the case of polarity reversal, however, the reversed field grows in the deep interior. Whenever the polarity changes in sequence of an excursion as well as a reversal, the reversed field appears in the northern and southern hemispheres.

\subsection{Internal process during transitional periods}

We now examine the internal structures of the magnetic and the velocity fields as well as the magnetic field generation processes responsible for the polarity transitions mentioned above. The magnetic induction term in Eq. (2) can be decomposed into the field advection term and the field stretching term with the divergence-free property of the magnetic and velocity fields:

$$
\nabla \times(\mathbf{u} \times \mathbf{B})=-(\mathbf{u} \cdot \nabla) \mathbf{B}+(\mathbf{B} \cdot \nabla) \mathbf{u} .
$$

Distributions of the radial component of the magnetic field, the velocity field, the advection term, and the stretching term at the radii, $r=r_{1}=r_{o}-0.009$ and $r=r_{2}=r_{i}+0.5$ (mid-depth), viewed from the south are plotted, in Fig. 3, at around the start and end times of the $\mathrm{R} 1$ event. At $t=$ 0.735 , the positive $B_{r}$ prevails at mid- to high-latitudes, and strong spots correlate with the down-welling flow associated with the edge of very narrow convection columns at $r=r_{1}$. Also, there are some areas with negative $B_{r}$ at midto high-latitudes without strong spots. Positive $B_{r}$ regions show some correlation with the strongly speckled regions of the down-welling flow, the advection, and the stretching terms, whereas the negative $B_{r}$ regions have no obvious correlation with any of them, suggesting that negative $B_{r}$ is temporally unstable there if this situation persists. At the same time, some negative $B_{r}$ spots, corresponding to the reversed field shown in Fig. 2, as well as the positive spots appear at $r=r_{2}$. The negative $B_{r}$ is generated by the stretching process between the convection cells. Positive correlation between the advection and the stretching terms is seen at some places, suggesting that the negative $B_{r}$ is advected outward together with intensification by the stretching process.

The polarity at $r=r_{1}$ has reversed at $t=0.755$. The strong negative $B_{r}$ regions show some spatial correlation with the speckled advection and the stretching terms, which is not found in the negative $B_{r}$ regions at $t=0.735$. It is thus suggested that correlation with these terms is important to 

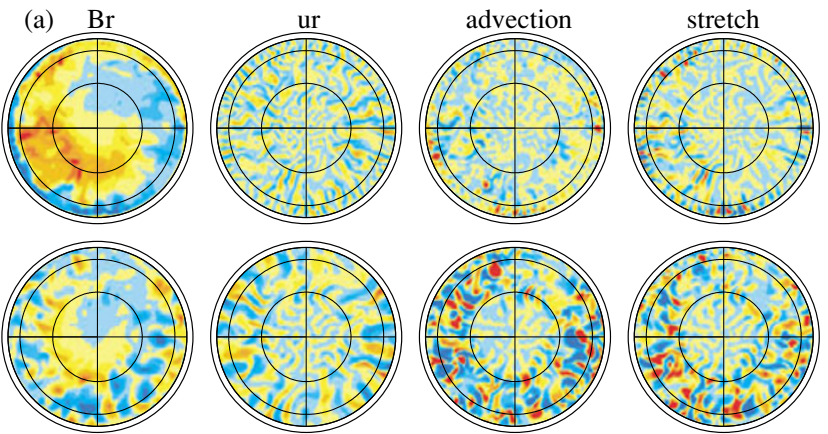

(b)
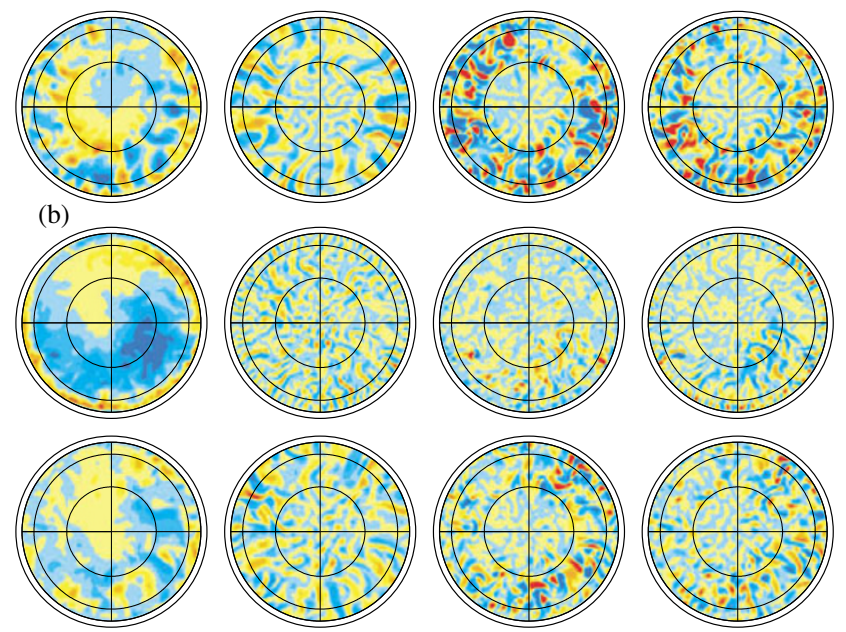

Fig. 3. Distribution of the radial component of the magnetic field, the velocity field, the advection, and the stretching terms in the induction equation at (a) $t=0.73500$ and (b) $t=0.75500$ in $\mathrm{R} 1$ event viewed from the south. The top panel is at $r=r_{1}=r_{o}-0.009$, while the bottom panel is at $r=r_{2}=r_{i}+0.5$. Contour interval is $0.3(0.04)$ for $B_{r}, 120$ (20) for $u_{r}$, and 400 (160) for the advection and stretching terms at $r=r_{2}\left(r_{1}\right)$. Red (blue) regions represent positive (negative) values.

maintain the new polarity at the core surface. Although this correlation is likely present somewhere also in E1 and E4, the reversed polarity is not sustained when inverse field generation is inactive. In the $\mathrm{R} 1$ event, the modest correlation with active inverse field generation lasts for a sufficiently long time, and the negative radial component is collected by the down-welling flow associated with columnar convective motions near the core surface, as usually found in dynamo models with stable polarity (e.g. Olson et al., 1999).

The duration of the reversal sequence is about 0.006 viscous diffusion times (Fig. 1), whereas the field advection time, from the deep to shallow interiors, in the radial direction is roughly estimated to be 0.015 , as partly shown in Fig. 2. This is longer than the estimate of half the shell turnover time, $L / u \sim 0.003$ with $u \sim 300$, because the advection path meanders due to the complicated spatial structure of convection, as shown in the next subsection. The field propagation time from the southern to the northern hemisphere is about 0.01 , from 0.745 to 0.755 , as shown in Fig. 2, which is comparable to the estimate of half the meridional advection time, $0.5 \pi r_{o} / u \sim 0.008$. It is therefore likely that the reversed field is transported in the longitudinal direction by advection. The reversal process seems to be consistent with that by Wicht and Olson (2004). It should be noted, however, that the typical velocity to estimate the advection time is the root-mean-square velocity in the shell, whereas the meridional flow is responsible for advection in Wicht and Olson's model. In the present model, the meridional flow component contains not more than $0.1 \%$ of the kinetic energy.
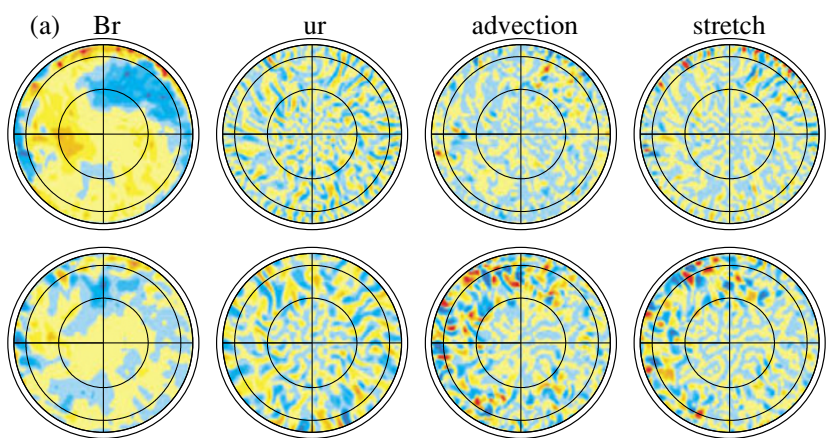

(b)
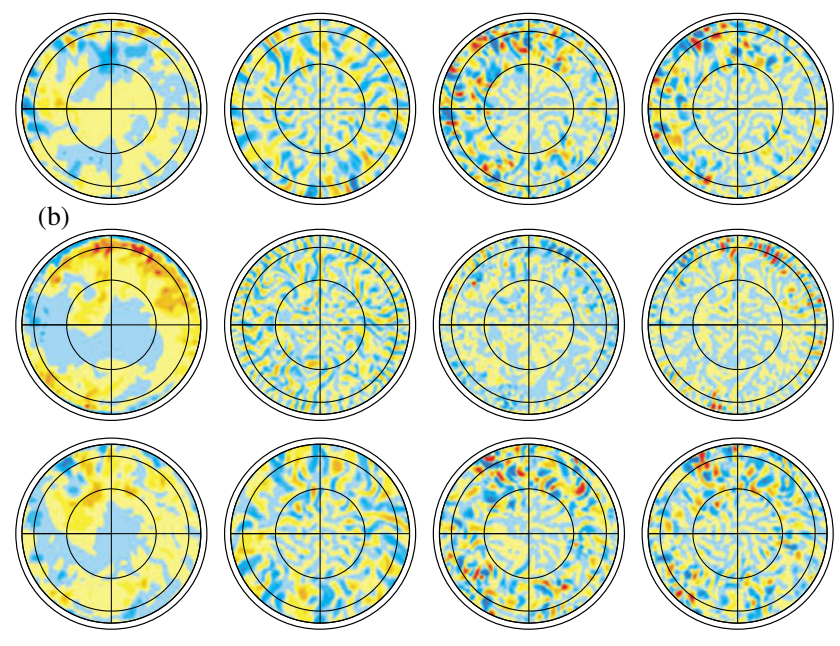

Fig. 4. Same as Fig. 3 but at (a) $t=0.56500$ and (b) $t=0.58500$ in E1 event.
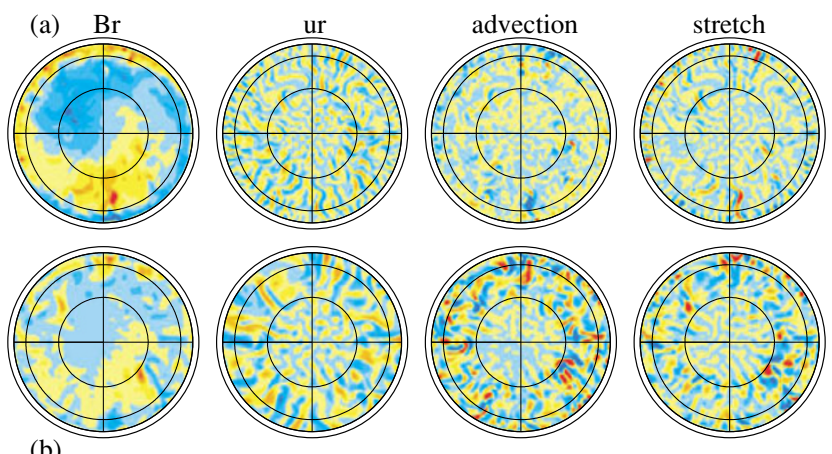

(b)
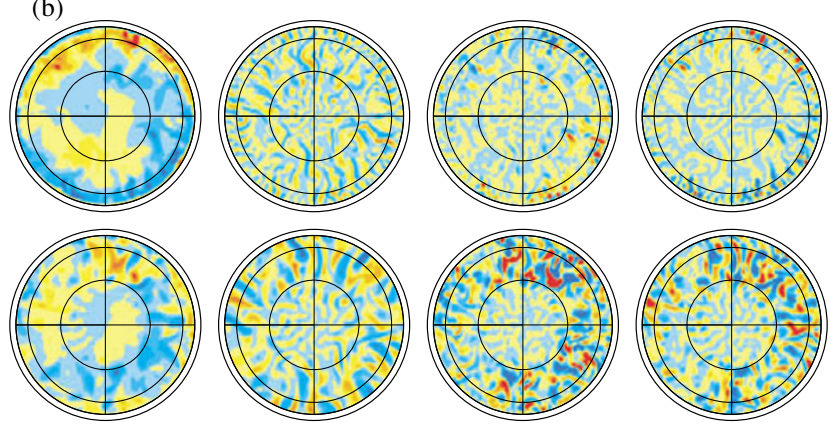

Fig. 5. Same as Fig. 3 but at (a) $t=0.95500$ and (b) $t=0.96500$ in E4 event.

The same plots as those shown in Fig. 3, but for the E1 and E4 events, are displayed in Figs. 4 and 5, respectively. In Fig. 4(a), there are some negative (reversed) fields at mid- to high-latitudes at $r=r_{1}$. However, it seems that correlation with the advection and the stretching terms is very poor. Also, the amplitudes of the advection and the stretching terms are small compared with those in Fig. 3. Then, the positive (normal) field is soon retrieved due to the stretching process deep in the core, as in Fig. 4(b). After the normal magnetic field reaches the core surface at low- 
Table 2. Symmetry of the magnetic field induced by each interaction.

\begin{tabular}{l|ll}
\hline & $B^{S}$ & $B^{A}$ \\
\hline$u^{S}$ & $B^{S}$ & $B^{A}$ \\
$u^{A}$ & $B^{A}$ & $B^{S}$ \\
\hline
\end{tabular}
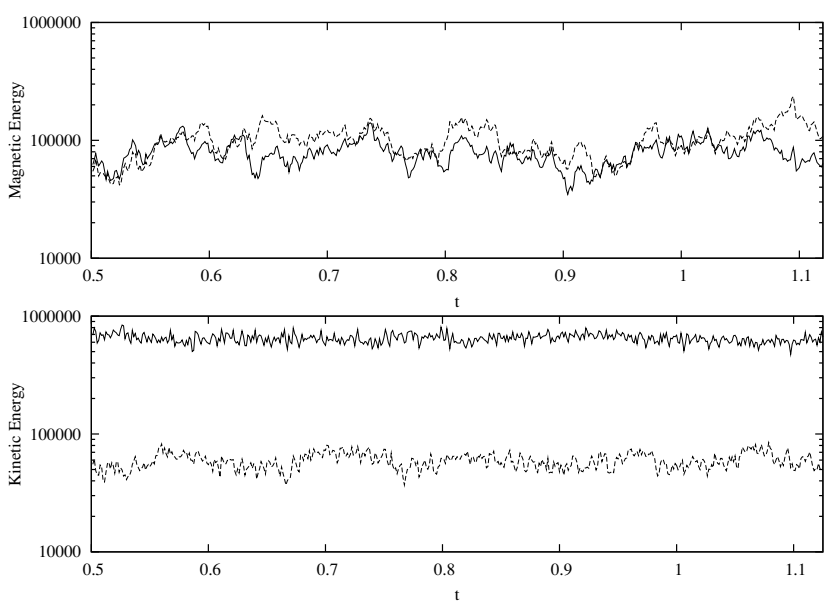

Fig. 6. Time series of the magnetic (top) and kinetic (bottom) energy density in the core. Solid (dashed) lines denote the equatorially symmetric (anti-symmetric) component. The magnetic energy is re-scaled by multiplying a factor $(E P m)^{-1}$ so as to make direct comparison with the kinetic energy possible.

latitudes, it is propagated to higher latitudes. A similar process is found in the northern hemisphere. After all, the normal polarity survives. In the E4, what is happening in the southern hemisphere is similar to the E1 (Fig. 5). As noted above, the dipole component is weak during events E4-E6. Thus, the magnetic pole can easily be disturbed even by the weak reversed field.

On the whole, the magnetic field shows a dipole-familydominated, or an equatorially anti-symmetric (EA) structure, while the velocity field shows an equatorially symmetric (ES) structure due to the effects of rapid rotation. The poloidal field in dipole (quadrupole) family consists of the components with $\ell-m$ odd (even), while the families interchange in the toroidal field with the same $\ell$ and $m$. Dynamo action allows four interactions between the velocity and magnetic field components in each family, as summarized in Table 2. If the magnetic and velocity fields belong to purely EA and ES families, respectively, the interaction between these fields regenerates the purely EA family magnetic field because of the symmetry property of the induction equation (Gubbins and Zhang, 1993). However, these symmetries are apparently broken during transitional periods. We decompose the velocity and magnetic fields into EA and ES families

$$
\begin{aligned}
\mathbf{u} & =\mathbf{u}^{A}+\mathbf{u}^{S}, \\
\mathbf{B} & =\mathbf{B}^{A}+\mathbf{B}^{S},
\end{aligned}
$$

where superscripts $A$ and $S$ denote EA and ES family components, respectively, and then we analyze the behavior of each family. Figure 6 shows the time series of the magnetic and kinetic energy in each family. It is evident that the magnetic energy in both families is comparable to each

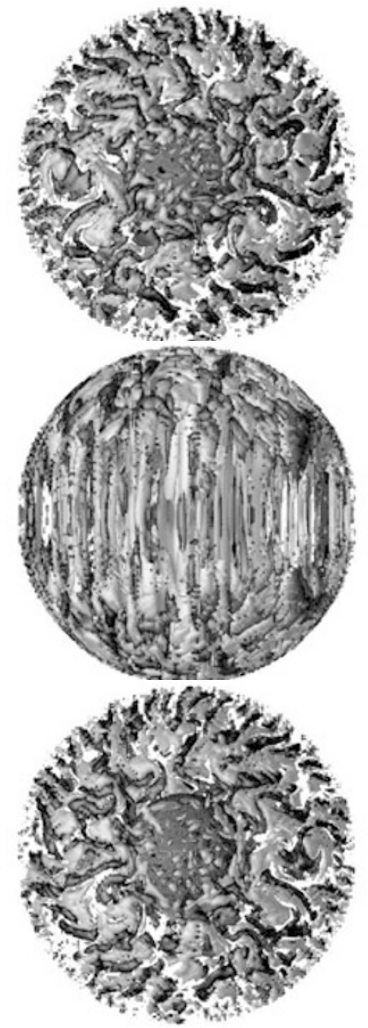

Fig. 7. 3-D views of the convection structure. Drawn are isosurfaces of the axial component of the vorticity $\left(\left|\omega_{z}\right|=6000\right)$ viewed from the north (top), equator (middle) and south (bottom). Positive (negative) value is represented in black (gray).

other, whereas the kinetic energy in the ES family is dominant; the kinetic energy in ES family is ten-fold larger than that in the EA family. This fact suggests that $\mathbf{u}^{S}$, whose main constituent is columnar convection vortices, is most responsible for dynamo action. Figure 6 suggests that $\mathbf{B}^{A}$ is generated primarily by the interaction between $\mathbf{u}^{S}$ and $\mathbf{B}^{A}$, while $\mathbf{B}^{S}$ is generated by the interaction between $\mathbf{u}^{S}$ and $\mathbf{B}^{S}$. The correlation coefficient between time series of the magnetic energy of $\mathbf{B}^{A}$ and $\mathbf{B}^{S}$ is 0.59 . The modest correlation does not deny that $\mathbf{B}^{A}$ and $\mathbf{B}^{S}$ are maintained primarily by $\mathbf{u}^{S}$.

\subsection{Spatial structures of convection and magnetic field}

A 3-D plot is very helpful to understand the complicated structures of the flow and the magnetic field. Displayed in Fig. 7 are snapshots of the 3-D structure of the axial component of the vorticity, $\omega_{z}=(\nabla \times \mathbf{u})_{z}$, north-polar, southpolar, and side view, visualized by isosurface rendering. It is evident that the fundamental flow structure outside the TC is characterized by the well-organized columnar flow nearly invariant in the axial direction and that the ES flow component is dominant. The shape of convective columns is radially and azimuthally deformed due to the large Rayleigh number. The flow structure inside the TC is characterized by the smaller scale components.

We next focus on the structure of the velocity and magnetic fields during the polarity reversal. Figure 8 shows snapshots of $\omega_{z}$ and $B_{z}$ during evolution of the reversal at $t=0.74125$. The 3-D distribution of helicity, $h=$ $\mathbf{u} \cdot(\nabla \times \mathbf{u})$, as exhibited in Fig. 8(a), obviously shows a 

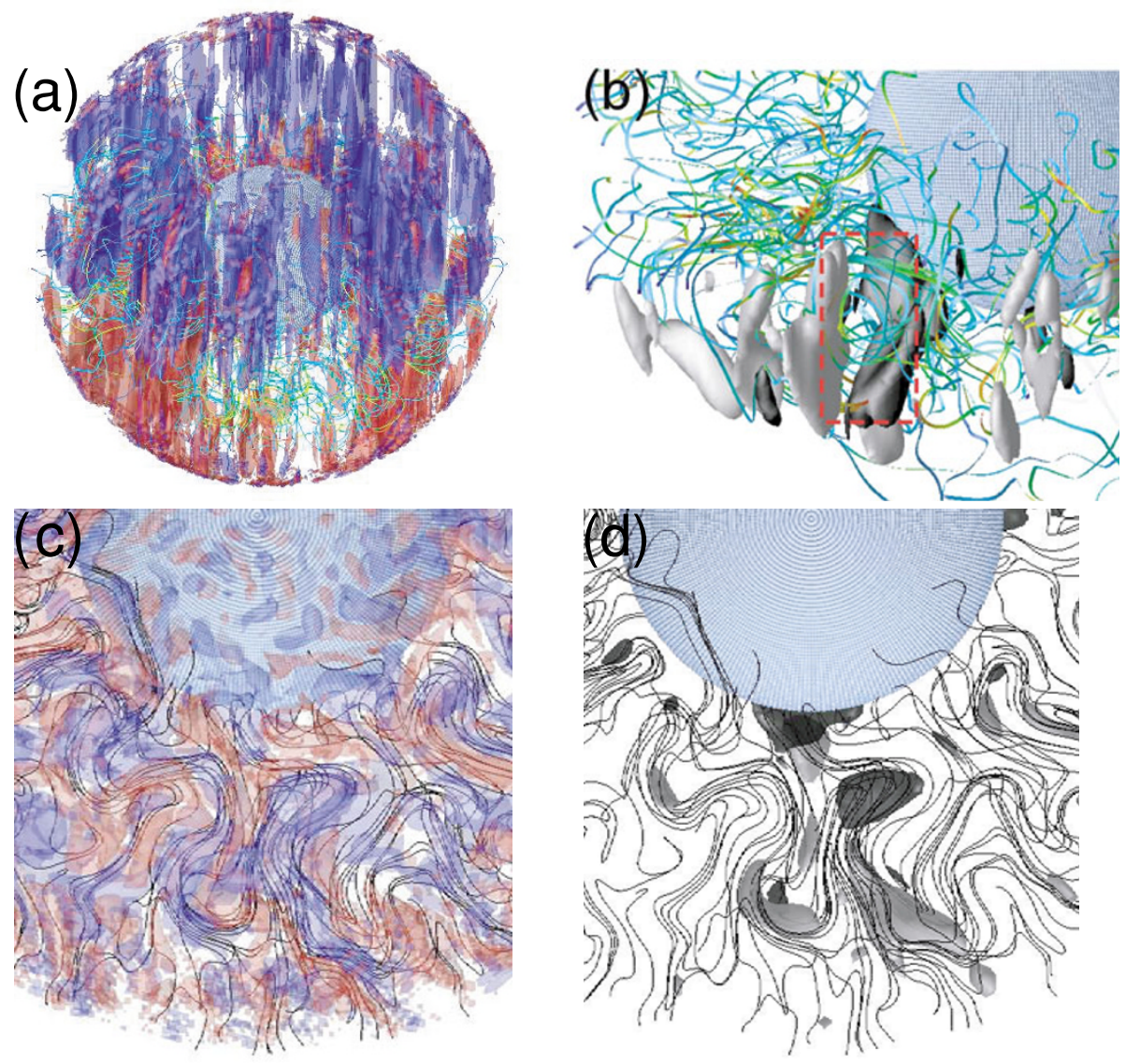

Fig. 8. Bird's-eye views of the magnetic lines of force at $t=0.74125$. Superimposed are (a): iso-surfaces of the helicity, $|h|=2 \times 10^{6}$, (b) and (d): iso-surfaces of the axial component of the magnetic field, $\left|B_{z}\right|=1.0$, (c): iso-surfaces of the axial vorticity, $\left|\omega_{z}\right|=6000$. (b) is viewed from the equator, while (c) and (d) are viewed from the north. Positive (negative) $B_{z}$ is represented by the black (gray) blob, while positive (negative) $\omega_{z}$ and $h$ are represented by transparent red (blue). The solid inner core is represented by the light-blue sphere. In (a) and (b) magnetic field strength is described by means of color. Blue lines denote weak magnetic field, and red lines denote strong magnetic field. The magnetic field loop remarked in the text is enclosed by the red square in (b).

well-known feature; that is, $h$ is predominantly negative in the northern hemisphere and positive in the southern hemisphere because of the columnar structure of the flow (Olson et al., 1999). This also suggests that the columnar flow is responsible for this dynamo process. Helicity with a sign opposing the predominant helicity in either hemisphere is only found in limited regions near the outer boundary and is thus rendered unimportant for the dynamo process.

The magnetic field structure is represented in terms of the magnetic lines of force and isosurface of the axial component in Fig. 8(b)-(d). The magnetic lines of force look like entangled thread as a result of multiple bending in the directions perpendicular and parallel to the equatorial plane by the deformed convection vortices, as in Fig. 7. The strong positive $B_{z}$ appears near the inner core, whereas the negative $B_{z}$ appears in the outer portion. This structure is reflected in the axisymmetric field in Fig. 2. The positive and negative $B_{z}$ forms the most intense reversed field, or a magnetic loop structure perpendicular to the equatorial plane (Fig. 8(b)). When the field lines are seen from the north (Fig. 8(c), (d)), it is clearly found that the curvature of the magnetic lines of force corresponds well to the shape of convection columns and that the axial magnetic field is mostly intensified in the columns with negative $\omega_{z}$ (anticyclone). The magnetic loop is generated by the deformed convection columns, incident to highly active convection. Such a loop structure is never found during other events including stable periods.

Active convection inside the TC can cause a break in equatorial symmetry (Christensen et al., 1999). Takahashi et al. (2003) pointed out, however, that the dynamo action inside the TC plays a minor role for the polarity transition, since the magnetic field generated inside the TC is insignificant because of small-scale and highly time-dependent convection there. We conclude that polarity reversals are initiated at low-latitudes outside the TC, as found in this paper as well as by Takahashi et al. (2005).

\section{Discussion}

Using a Takahashi-Matsushima-Honkura dynamo model, we have investigated the internal process of dynamo action during some transitional periods. Although it is difficult to find a typical magnetic field generation process responsible for a polarity transition because of the very complicated 


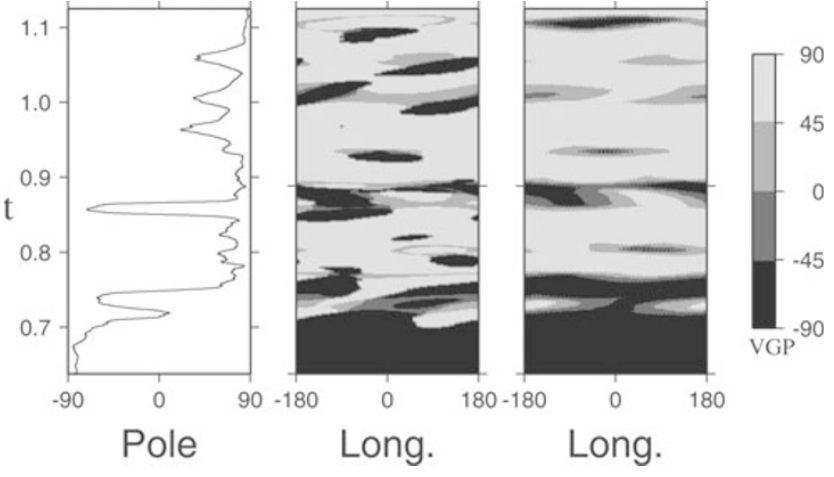

Fig. 9. Site dependence of the VGP latitude. The left plot is the time series of the true magnetic pole, whereas the middle (right) represents the time-longitude diagram of the VGP at $30^{\circ} \mathrm{N}\left(60^{\circ} \mathrm{N}\right)$.

structure, spatial, and temporal variations of the advection and stretching processes as well as the correlation among them seem to give rise to distinct differences in behavior of the magnetic field during a transitional period. The reversed field generated near the inner core at low-latitudes in one hemisphere moves toward the core surface, and then it is propagated toward higher-latitudes by advection. This process eventually gives rise to a polarity reversal. The sequence in our model is dynamically similar to that in the model by Wicht and Olson (2004). That is, the reversed field is transported by convective motions. The notable difference arises from the type of flow responsible for advection. In Wicht and Olson's model, a relatively high Ekman number may intensify the effect of EA flow, and thereby the axisymmetric meridional circulation advects the reversed flux from south to north. In the present model, however, the non-axisymmetric wavy convection columns due to a lower Ekman number play an important role in advection of the reversed field. As for magnetic field generation in terms of equatorial symmetry, the flow component in ES family, in particular, columnar convection vortices, seems to maintain the magnetic field in both ES and EA families. To what extent the EA flow is important to the generation of the the reversed magnetic field in the present model is not obvious, but the sequence of polarity reversal similar to Wicht and Olson's model suggests that the EA flow influences the polarity transition. As a possible scenario, the reversed field might initially be generated by the EA flow, following which it is enhanced by the ES flow. It is obviously the next step to examine how the EA flow affects dynamo action.

From a paleomagnetic point of view, it is known that the duration of polarity transition shows site-dependence (Clement, 2004), and its north-south asymmetry (Wicht, 2005), which is large for reversals and relatively small for excursions, with polarity change. A closer comparison with paleomagnetic data is necessary to assess the validity of different simulated reversals. Figure 9 shows the timelongitude diagrams of VGP latitude, which indicate that VGPs basically reflect the true dipole signatures. However, a short polarity event is also observed at low-latitudes during excursions (E4-E6). Furthermore, the non-dipole signatures give rise to local (not global) excursions, and lon- gitudinal dependence appears on several occasions. These facts suggest that a good spatial coverage of paleomagnetic data during a transitional period is essential to restore the global paleomagnetic field.

In the present paper, attention is exclusively paid to the induction process, and thus one may argue that the back reaction from the magnetic field to the velocity field should be investigated. We consider that the magnetic field in the model is not strong enough to significantly affect the velocity field and that polarity reversals are nearly free from the Lorentz force, as in the model by Wicht and Olson (2004).

Unfortunately, the present analysis is based on a few samples. Coe et al. (2000) remark that polarity reversals take place in various ways. For example, Sarson and Jones (1999) show that reversals in their model are triggered by the intermittent flow inside the TC. Undoubtedly, more reversal processes should be analyzed with the aim of understanding of the polarity reversal mechanisms.

\section{Conclusions}

We summarize the conclusions of the present paper in the following.

1. There are distinctive differences in the behavior of the magnetic field during transitional periods due to the spatial and temporal variations of the advection and stretching processes. At the beginning of polarity transitions, the magnetic field with the reversed polarity is generated at low-latitudes in the deep interior of the outer core. The reversed flux is advected outward and is also propagated toward high-latitudes. The polarity reversal is completed by the advection process. In the case of excursion, however, the reversed field is not generated successively, and the original polarity is retrieved.

2. The radially and azimuthally deformed convection columns generate the reversed field, or the magnetic loop perpendicular to the equatorial plane, which is intensified by the convective motion in anti-cyclones. The magnetic loop eventually causes the polarity reversal.

3. Duration of the field advection from the deep to shallow interiors is longer than a simple estimate of half the shell turnover time because of the complicated spatial structure of convection. Duration of the reversed field propagation from one to the other hemisphere near the core surface is comparable to the estimate of half the meridional advection time.

4. Columnar convection vortices are dominant and responsible for generating the magnetic fields in both the equatorially symmetric and equatorially antisymmetric families. This suggests that the magnetic field is regenerated primarily from the magnetic field that belongs to the same family.

Acknowledgments. Numerical simulation was carried out on the Earth Simulator under the support of JAMSTEC. The authors are very grateful to Robert S. Coe and Johannes Wicht for their useful comments and constructive discussion, which substantially improved the manuscript. Futoshi Takahashi was supported by 
Research Fellowships of the Japan Society for the Promotion of Science for young scientists. This work is partially supported by the Japan Society for the Promotion of Science under the grant-inaid for scientific research No. 18340131.

\section{References}

Bouligand, C., G. Hulot, A. Khokhlov, and G. A. Glatzmaier, Statistical palaeomagnetic field modelling and dynamo numerical simulation, Geophys. J. Int., 161, 603-626, 2005.

Brown, L. L., B. S. Singer, J. C. Pickens, and B. R. Jicha, Paleomagnetic directions and ${ }^{40} \mathrm{Ar} /{ }^{39} \mathrm{Ar}$ ages from the Tatara-San Pedro volcanic complex, Chilean Andes: Lava record of a Matuyama-Brunhes precursor?, J. Geophys. Res., 109, B12101, doi:10.1029/2004JB003007, 2004.

Christensen, U. R. and J. Aubert, Scaling properties of convection-driven dynamos in rotating spherical shells and application to planetary magnetic fields, Geophys. J. Int., 166, 97-114, 2006.

Christensen, U. R., P. Olson, and G. A. Glatzmaier, Numerical modelling of the geodynamo: A systematic parameter study, Geophys. J. Int., 138, 393-409, 1999.

Clement, B. M., Dependence of the duration of geomagnetic polarity reversals on site latitude, Nature, 428, 637-640, 2004.

Coe, R., L. Hongre, and G. A. Glatzmaier, An examination of simulated geomagnetic reversals from a palaeomagnetic perspective, Philos. Trans. R. Soc. Lond. A, 358, 1141-1170, 2000.

Glatzmaier, G. A. and P. H. Roberts, A three-dimensional self-consistent computer simulation of a geomagnetic field reversal, Nature, 377, 203209, 1995.

Gubbins, D., The distinction between geomagnetic excursions and reversals, Geophys. J. Int., 137, F1-F4, 1999.

Gubbins, D. and K. Zhang, Symmetry properties of the dynamo equations for palaeomagnetism and geomagnetism, Phys. Earth Planet. Inter., 75, 225-241, 1993.

Guillou, H., B. S. Singer, C. Laj, C. Kissel, S. Scaillet, and B. R. Jicha, On the age of the Laschamp geomagnetic excursion, Earth Planet. Sci. Lett, 227, 331-343, 2004.

Kageyama, A., T. Sato, and the Complexity Simulation Group, Computer simulation of a magnetohydrodynamic dynamo II, Phys. Plasmas, 2, 1421-1431, 1995.

Kono, M. and P. H. Roberts, Recent geodynamo simulations and observations of the geomagnetic field, Rev. Geophys., 40, 1013, doi:10. 1029/2000RG000102, 2002.

Kutzner, C. and U. R. Christensen, From stable dipolar towards reversing numerical dynamos, Phys. Earth Planet. Inter., 131, 29-45, 2002.

Kutzner, C. and U. R. Christensen, Simulated geomagnetic reversals and preferred virtual geomagnetic pole paths, Geophys. J. Int., 157, 1105$1118,2004$.

Li, J., T. Sato, and A. Kageyama, Repeated and sudden reversals of the dipole field generated by a spherical dynamo action, Science, 295, 1887-1890, 2002.

McMillan, D. G., C. G. Constable, R. L. Parker, and G. A. Glatzmaier, A statistical analysis of magnetic fields from some geodynamo simulations, Geochem. Geophys. Geosyst., 2, doi:10.1029/2000GC000130, 2001.
Merrill, R., M. McElhinny, and P. McFadden, The Magnetic Field of the Earth, Academic Press, San Diego, 1996.

Mochizuki, N., H. Tsunakawa, H. Shibuya, T. Tagami, A. Ozawa, J. Cassidy, and I. E. M. Smith, K-Ar ages of the Auckland geomagnetic excursions, Earth Planets Space, 56, 283-288, 2004.

Mochizuki, N., H. Tsunakawa, H. Shibuya, J. Cassidy, and I. E. M. Smith, Palaeointensities of the Auckland geomagnetic excursions by the LTDDHT Shaw method, Phys. Earth Planet. Inter., 154, 168-179, 2006.

Mochizuki, N., H. Tsunakawa, H. Shibuya, T. Tagami, A. Ozawa, and I. E. M. Smith, Further K-Ar dating and paleomagnetic study of the Auckland geomagnetic excursions, Earth Planets Space, 59, this issue, 755-761, 2007.

Oda, H., M. J. Dekkers, C. G. Langereis, L. Lourens, and D. Heslop, A paleomagnetic record of the last $640 \mathrm{kyr}$ from an eastern Mediterranean piston core and a review of geomagnetic excursions in the Brunhes, EOS Trans. AGU, 95, GP41B-08 (Abstract), 2004.

Olson, P. and U. R. Christensen, Dipole moment scaling for convectiondriven planetary dynamos, Earth Planet. Sci. Lett, 250, 561-571, 2006.

Olson, P., U. R. Christensen, and G. A. Glatzmaier, Numerical modeling of the geodynamo: Mechanisms of field generation and equilibration, $J$. Geophys. Res., 104, 10383-10404, 1999.

Sarson, G. R. and C. A. Jones, A convection driven geodynamo reversal model, Phys. Earth Planet. Inter., 111, 3-20, 1999.

Singer, B. S., M. K. Relle, K. A. Hoffman, A. Battle, C. Laj, H. Guillou, and J. C. Carracedo, Ar/Ar ages from transitionally magnetized lavas on La Palma, Canary Islands, and the geomagnetic instability timescale, $J$. Geophys. Res., 107, 2307, doi:10.1029/2001JB001613, 2002.

Takahashi, F. and M. Matsushima, Dynamo action in a rotating spherical shell at high Rayleigh numbers, Phys. Fluids, 17, 076601, 2005.

Takahashi, F., J. S. Katayama, M. Matsushima, and Y. Honkura, Effects of boundary layers on magnetic field behavior in an MHD dynamo model, Phys. Earth Planet. Inter., 128, 149-161, 2001.

Takahashi, F., M. Matsushima, and Y. Honkura, Dynamo action and its temporal variation inside the tangent cylinder in MHD dynamo simulations, Phys. Earth Planet. Inter., 140, 53-71, 2003.

Takahashi, F., M. Matsushima, and Y. Honkura, Simulations of a quasiTaylor state geomagnetic field including polarity reversals on the Earth Simulator, Science, 309, 459-461, 2005.

Takahashi, F., M. Matsushima, and Y. Honkura, Scale variability in convection-driven MHD dynamos at low Ekman number, Geophys. J. Int., 2007 (submitted).

Wicht, J., Inner-core conductivity in numerical dynamo simulations, Phys. Earth Planet. Inter., 132, 281-302, 2002.

Wicht, J., Palaeomagnetic interpretation of dynamo simulations, Geophys. J. Int., 162, 371-380, 2005.

Wicht, J. and P. Olson, A detailed study of the polarity reversal mechanism in a numerical dynamo model, Geochem. Geophys. Geosyst., 5, Q03H10, doi:10.1029/2003GC000602, 2004.

F. Takahashi (e-mail: futoshi@geo.titech.ac.jp), M. Matsushima, and Y. Honkura 